\title{
A mouse is a mouse is a mouse
}

\author{
The increasing human mimicry and sophistication of organ chips and organoids bring into \\ question the demand for small animal models in biomedical research.
}

In March 2021, researchers reported for the first time lab-grown cellular models of human blastocysts, formed by guided self-organization of human cells. These blastoids not only mark an important step towards the modelling of human development but also highlight the power of in vitro platforms for the design of human-based models.

Organoids have already been developed for a variety of tissues, based on the same principle of cellular selforganization, with the aim of creating human models for the investigation of human (patho)physiology, and for the preclinical testing of therapies and drugs. Similarly, microfluidic human organs-on-a-chip have been designed, which, for some organs, can fully mimic organ function and interactions. Such in vitro models have, for example, been successfully applied in COVID-19 research, where animal models were not readily available and indeed, not always adequate, as discussed in a Comment in this issue. In an In Brief in this issue, we also highlight how human cardiac organoids enabled the identification of the mechanisms underlying cardiac injury and dysfunction in patients with COVID-19.

The obvious advantage of human in vitro models is that they are human, and thus, relevant for testing human conditions and biology. However, they lack the complexity of an organism. Therefore, small animal models, in particular mice, remain state-of-the-art for drug testing and fundamental biomedical research. We know, however, that many phenotypes observed in mice do not necessarily reflect the underlying biological mechanisms in humans. This discrepancy can not only lead to clinical failures but can also result in the wrong interpretation of biological and disease mechanisms. In addition, there are ethical concerns when it comes to the use of animals in biomedical research. However, scientific tradition, the possibility of genetic engineering, regulatory demand and a lack of organism-level alternatives have established small animal models as the holy grail of preclinical research.
Talking to Donald Ingber, who has developed and worked for many years with organs-on-chip platforms, we wondered why, despite great advances in organ chip and organoid engineering, many researchers remain reluctant to use human-based in vitro models, in particular in cases, in which they may be more adequate than animal models. For example, investigating how breathing motion influences the rate of human lung cancer growth and invasion cannot be done in animal models, for obvious reasons. Human lung chips not only enable such studies but also allow the controlled delineation of factors impacting cellular behaviour and fate. In an In Brief in this issue, we discuss lung chips based on stretchable biological membranes. These lung chips can functionally replicate breathing motion and the alveolar network of lungs. Of note, genetic engineering and the implementation of immune system components are also possible in such in vitro models.

Materials scientists and engineers have greatly contributed to the progress in human-based in vitro models, for example, by designing materials that can reproducibly guide organoid growth and morphogenesis, by engineering microfluidic platforms and optimizing biofabrication techniques for the design of organ-on-a-chip platforms and by developing multiplexed sensors to improve functional readouts.

So, do you have to be an engineer or materials scientist to use and apply such systems in your research? Certainly not. Organoids may be tricky to develop and culture. But it may take less time and effort to learn how to work with organ chips (some of which are already commercially available) than with mice. An open mind will be required from researchers, funding and regulatory agencies, and editors, to assess the most appropriate model system for a given experiment. Whether this is a mouse, a chip, an organoid, or a combination thereof, will depend on the biological and/or biomedical question asked. But it is not always a mouse. 